\title{
OPTIMAL CASORATI INEQUALITIES ON BI-SLANT SUBMANIFOLDS OF GENERALIZED SASAKIAN SPACE FORMS
}

\author{
ALIYA NAAZ SIDDIQUI
}

\begin{abstract}
In this paper, we use T Oprea's optimization method to establish some optimal Casorati inequalities, which involve the normalized scalar curvature for bi-slant submanifolds of generalized Sasakian space forms. In the continuation, we show that in both cases, the equalities hold if and only if submanifolds are invariantly quasi-umbilical.
\end{abstract}

\section{Introduction}

B. Y. Chen [5] introduced the new types of Riemannian invariants, known in the literature as Chen invariants and obtained general optimal inequalities consisting of the new intrinsic invariants and the main extrinsic invariants for any Riemannian submanifolds. It was the starting point of the theory of Chen invariants, which are one of the most interesting research topics in differential geometry $[11,12,13,14,16,17,18,19,24]$. Instead of concentrating on the sectional curvature with the extrinsic squared mean curvature, the Casorati curvature of a submanifold in a Riemannian manifold was considered as an extrinsic invariant defined as the normalized square of the length of the second fundamental form. The notion of Casorati curvature extends the concept of the principal direction of a hypersurface of a Riemannian manifold. Several geometers in $[6,7,10,20,21]$ found geometrical meaning and the importance of the Casorati curvature. Therefore, it is of great interest to obtain optimal inequalities for the Casorati curvatures of submanifolds in different ambient spaces. As a natural prolongation of our research, in the present paper we will study these inequalities for bi-slant submanifolds in generalized Sasakian space forms by using T Oprea's optimization method.

Our work is structured as follows: Section 2 is basically devoted to preliminary part, in which we summarize some basic material about generalized Sasakian space form including its Riemannian curvature tensor. In Section 3, we give proof of the main theorem of this paper

Received October 29, 2017, accpted April 30, 2018.

2010 Mathematics Subject Classification. 53B05, 53C25, 53B20, 53C40.

Key words and phrases. Casorati curvature, bi-slant submanifold, generalized Sasakian space form, quasi-umbilical submanifold. 
(Theorem 3.1). The purpose of this paper is to give another proof of optimal Casorati inequalities, which have been derived by A. N. Siddiqui and M. H. Shahid in [16], by using T Oprea's optimization method.

\section{Preliminaries}

A $(2 m+1)$-dimensional differentiable manifold $\overline{\mathscr{M}}$ is said to have an almost contact structure $(\phi, \xi, \eta, g)$ if there exists on $\overline{\mathscr{M}}$ a tensor field $\phi$ of type $(1,1)$, a vector field $\xi$, a 1 -form $\eta$ and a Riemannian metric $g$ such that [23]

$$
\begin{gathered}
\phi^{2}=-I+\eta \otimes \xi, \quad \phi \xi=0, \quad \eta(\xi)=1, \quad \eta(\phi)=0, \quad \eta(X)=g(X, \xi) \\
g(\phi X, \phi Y)=g(X, Y)-\eta(X) \eta(Y), \quad g(\phi X, Y)+g(X, \phi Y)=0
\end{gathered}
$$

Here $X, Y, Z$ denote arbitrary vector fields on $\overline{\mathscr{M}}$. The fundamental 2-form $\varphi$ on $\overline{\mathscr{M}}$ is defined by

$$
\varphi(X, Y)=g(\phi X, Y)
$$

Alegre et al. [1] introduced and studied the generalized Sasakian space forms. An almost contact metric manifold $(\overline{\mathscr{M}}, \phi, \xi, \eta, g)$ is said to be a generalized Sasakian space form if there exist differentiable functions $f_{1}, f_{2}, f_{3}$ such that curvature tensor $R$ of $\overline{\mathscr{M}}$ is given by

$$
\begin{aligned}
\bar{R}(X, Y) Z= & f_{1}[g(Y, Z) X-g(X, Z) Y]+f_{2}[g(X, \phi Z) \phi Y-g(Y, \phi Z) \phi X+2 g(X, \phi Y) \phi Z] \\
& +f_{3}[\eta(X) \eta(Z) Y-\eta(Y) \eta(Z) X+g(X, Z) \eta(Y) \xi-g(Y, Z) \eta(X) \xi]
\end{aligned}
$$

for all vector fields $X, Y, Z \in T \overline{\mathscr{M}}$.

Remark 1. The generalized Sasakian space form generalizes the concept of Sasakian space form, Kenmotsu space form and cosymplectic space form.

(i) A Sasakian space form is the generalized Sasakian space form with $f_{1}=\frac{c+3}{4}$ and $f_{2}=f_{3}=$ $\frac{c-1}{4}$.

(ii) A Kenmotsu space form is the generalized Sasakian space form with $f_{1}=\frac{c-3}{4}$ and $f_{2}=$ $f_{3}=\frac{c+1}{4}$.

(iii) A cosymplectic space form is the generalized Sasakian space form with $f_{1}=f_{2}=f_{3}=\frac{c}{4}$.

In the following we consider $\overline{\mathscr{M}}$ as a generalized Sasakian space form $\overline{\mathscr{M}}\left(f_{1}, f_{2}, f_{3}\right)$ of dimension $(2 m+1)$ and let $\mathscr{M}$ be an $(n+1)$-dimensional submanifold of $\overline{\mathscr{M}}\left(f_{1}, f_{2}, f_{3}\right)$. Let $T \mathscr{M}$ and $T^{\perp} \mathscr{M}$ denote the Lie algebra of vector fields and set of all normal vector fields on $\mathscr{M}$ 
respectively. The operator of covariant differentiation with respect to the Levi-Civita connection in $\mathscr{M}$ and $\overline{\mathscr{M}}$ is denoted by $\nabla$ and $\bar{\nabla}$, respectively. Let $\bar{R}$ and $R$ be the curvature tensor of $\overline{\mathscr{M}}\left(f_{1}, f_{2}, f_{3}\right)$ and $\mathscr{M}$, respectively. The Gauss equation is given by [23]

$$
\bar{R}(X, Y, Z, W)=R(X, Y, Z, W)-g(h(X, W), h(Y, Z))+g(h(X, Z), h(Y, W))
$$

for all vector fields $X, Y, Z \in T \overline{\mathscr{M}}$.

For any vector field $X \in T \mathscr{M}$, we put [23]

$$
\phi X=P X+Q X,
$$

where $P X$ and $Q X$ denote the tangential and normal components of $\phi X$, respectively. Then $P$ is an endomorphism of $T \mathscr{M}$, and $Q$ is the normal bundle valued 1-form on $T \mathscr{M}$.

In the same way, for any vector field $V \in T^{\perp} \mathscr{M}$, we put [23]

$$
\phi V=B V+C V,
$$

where $B V$ and $C V$ denote tangential and normal components of $\phi V$, respectively.

It is easy to see that $Q$ and $B$ are skew-symmetric and

$$
g(Q X, V)=-g(X, B V)
$$

for any vector fields $X \in T \mathscr{M}$ and $V \in T^{\perp} \mathscr{M}$.

The structural vector field $\xi$ can be decomposed as

$$
\xi=\xi_{1}+\xi_{2}
$$

where $\xi_{1}$ and $\xi_{2}$ are the tangential and the normal components of $\xi$.

A submanifold $\mathscr{M}$ of an almost contact metric manifold $\overline{\mathscr{M}}$ is said to be invariant if $Q \equiv 0$, that is, $\phi X \in T \mathscr{M}$, and anti-invariant if $P \equiv 0$, that is, $\phi X \in T^{\perp} \mathscr{M}$, for any vector field $X \in T \mathscr{M}$.

There are some other important classes of submanifolds which are determined by the behavior of tangent bundle of the submanifold under the action of an almost contact metric structure $\phi$ of $\overline{\mathscr{M}}$ :

(i) A submanifold $\mathscr{M}$ of $\overline{\mathscr{M}}$ is called a contact CR-submanifold [22] of $\overline{\mathscr{M}}$ if there exists a differentiable distribution $D$ on $\mathscr{M}$ whose orthogonal complementary distribution $D^{\perp}$ is anti-invariant.

(ii) A submanifold $\mathscr{M}$ of $\overline{\mathscr{M}}$ is called a slant submanifold [3] of $\overline{\mathscr{M}}$ if, the angle between $\phi X$ and $T_{x} \mathscr{M}$ is constant for all $X \in T \mathscr{M}-\left\{\xi_{x}\right\}$ and $x \in \mathscr{M}$. 
(iii) A submanifold $\mathscr{M}$ of $\overline{\mathscr{M}}$ is called semi-slant submanifold [2] of $\overline{\mathscr{M}}$ if there exists a pair of orthogonal distributions $D$ and $D_{\theta}$ such that $D$ is invariant and $D_{\theta}$ is proper slant.

(iv) A submanifold $\mathscr{M}$ of $\overline{\mathscr{M}}$ is called hemi-slant submanifold (or pseudo-slant) [9] of $\overline{\mathscr{M}}$ if there exists a pair of orthogonal distributions $D^{\perp}$ and $D_{\theta}$ such that $D^{\perp}$ is anti-invariant and $D_{\theta}$ is proper slant.

Bi-slant submanifolds were first defined by A. Cariazo et al. in [2] as a generalization of $\mathrm{CR}$ and semi-slant submanifolds. Such submanifolds generalize complex, totally real, slant and hemi-slant submanifolds as well. Here we define a bi-slant submanifold of an almost contact metric manifold as follows:

Definition 1. A submanifold $\mathscr{M}$ of an almost contact metric manifold $\overline{\mathscr{M}}$ is said to be a $b i$ slant submanifold if there exists a pair of orthogonal distributions $D_{\theta_{1}}$ and $D_{\theta_{2}}$ of $\mathscr{M}$ such that

(i) $T \mathscr{M}=D_{\theta_{1}} \oplus D_{\theta_{2}} \oplus\{\xi\}$;

(ii) $\phi D_{\theta_{1}} \perp D_{\theta_{2}}$ and $\phi D_{\theta_{2}} \perp D_{\theta_{1}}$;

(iii) Each distribution $D_{\theta_{i}}$ is slant with the slant angle $\theta_{i}$ for $i=1,2$.

A bi-slant submanifold of an almost contact metric manifold $\overline{\mathscr{M}}$ is called proper if the slant distributions $D_{\theta_{1}}$ and $D_{\theta_{2}}$ are of the slant angles $\theta_{1}, \theta_{2} \neq 0, \frac{\pi}{2}$.

Suppose that $\mathscr{M}$ is a bi-slant submanifold of dimension $n+1=2 n_{1}+2 n_{2}+1$ in $\overline{\mathscr{M}}\left(f_{1}, f_{2}, f_{3}\right)$. Let us assume the orthonormal basis of $\mathscr{M}$ as follows :

$$
\begin{aligned}
E_{1}, E_{2} & =\sec \theta_{1} P e_{1}, \ldots, E_{2 n_{1}-1}, E_{2 n_{1}}=\sec \theta_{1} P e_{2 n_{1}-1}, E_{2 n_{1}+1}, E_{2 n_{1}+2} \\
& =\sec \theta_{2} P e_{2 n_{1}+1}, \ldots, E_{2 n_{1}+2 n_{2}-1}, E_{2 n_{1}+2 n_{2}}=\sec \theta_{2} P e_{2 n_{1}+2 n_{2}-1}, E_{2 n_{1}+2 n_{2}+1}=\xi .
\end{aligned}
$$

Also,

$$
g^{2}\left(\phi E_{i+1}, E_{i}\right)=\left\{\begin{array}{lll}
\cos ^{2} \theta_{1} & \text { for } & i=1, \ldots, 2 n_{1}-1 \\
\cos ^{2} \theta_{2} & \text { for } & i=2 n_{1}+1, \ldots, 2 n_{1}+2 n_{2}-1
\end{array}\right.
$$

Hence, we have

$$
\sum_{i, j=1}^{n+1} g^{2}\left(\phi E_{j}, E_{i}\right)=2\left\{n_{1} \cos ^{2} \theta_{1}+n_{2} \cos ^{2} \theta_{2}\right\}
$$

Remark 2. If we assume

(i) $\theta_{1}=0$ and $\theta_{2}=\frac{\pi}{2}$, then $\mathscr{M}$ is a CR-submanifold.

(ii) $\theta_{1}=0$ and $\theta_{2} \neq 0, \frac{\pi}{2}$, then $\mathscr{M}$ is a semi-slant submanifold.

(iii) $\theta_{1}=\frac{\pi}{2}$ and $\theta_{2} \neq 0, \frac{\pi}{2}$, then $\mathscr{M}$ is a hemi-slant submanifold. 


\section{An upper bound of normalized scalar curvature by using $\mathrm{T}$ Oprea's optimization method}

In this section, we obtain some optimal Casorati inequalities for $\mathscr{M}$ in $\overline{\mathscr{M}}\left(f_{1}, f_{2}, f_{3}\right)$. For this, we choose a local orthonormal tangent frame $\left\{E_{1}, \ldots, E_{n+1}\right\}$ of the tangent bundle $T \mathscr{M}$ of $\mathscr{M}$ and a local orthonormal normal frame $\left\{E_{n+2}, \ldots, E_{2 m+1}\right\}$ of the normal bundle $T^{\perp} \mathscr{M}$ of $\mathscr{M}$ in $\overline{\mathscr{M}}\left(f_{1}, f_{2}, f_{3}\right)$. At any $p \in \mathscr{M}$, the scalar curvature $\tau$ at that point is given by

$$
\tau=\sum_{1 \leq i<j \leq n+1} R\left(E_{i}, E_{j}, E_{j}, E_{i}\right)
$$

and the normalized scalar curvature $\rho$ of $M$ is defined as

$$
\rho=\frac{2 \tau}{n(n+1)} .
$$

The mean curvature vector denoted by $\mathscr{H}$ of $\mathscr{M}$ is given by

$$
\mathscr{H}=\sum_{i=1}^{n+1} \frac{1}{n+1} h\left(E_{i}, E_{i}\right)
$$

Conveniently, let us put

$$
h_{i j}^{r}=g\left(h\left(E_{i}, E_{j}\right), E_{r}\right)
$$

for $i, j=\{1, \ldots, n+1\}$ and $r=\{n+2, \ldots, 2 m+1\}$. Then the squared norm of mean curvature vector of $\mathscr{M}$ is defined as

$$
\|\mathscr{H}\|^{2}=\frac{1}{(n+1)^{2}} \sum_{r=n+2}^{2 m+1}\left\{\sum_{i=1}^{n+1} h_{i i}^{r}\right\}^{2} .
$$

and the squared norm of second fundamental form $h$ is denoted by

where

$$
\mathscr{C}=\frac{1}{n+1}\|h\|^{2},
$$

$$
\|h\|^{2}=\sum_{r=n+2}^{2 m+1} \sum_{i, j=1}^{n+1}\left(h_{i j}^{r}\right)^{2} .
$$

It is known as the Casorati curvature $\mathscr{C}$ of $\mathscr{M}$.

If we suppose that $\mathscr{L}$ is an $s$-dimensional subspace of $T \mathscr{M}, s \geq 2$, and $\left\{E_{1}, \ldots, E_{s}\right\}$ is an orthonormal basis of $\mathscr{L}$, then the scalar curvature of the $s$-plane section $\mathscr{L}$ is given by

$$
\tau(\mathscr{L})=\sum_{1 \leq i<j \leq s} R\left(E_{i}, E_{j}, E_{j}, E_{i}\right)
$$

and the Casorati curvature of the subspace $\mathscr{L}$ is as follows

$$
\mathscr{C}(\mathscr{L})=\frac{1}{s} \sum_{r=n+2}^{2 m+1} \sum_{i, j=1}^{s}\left(h_{i j}^{r}\right)^{2}
$$


The normalized $\delta$-Casorati curvatures $\delta_{\mathscr{C}}(n)$ and $\widehat{\delta}_{\mathscr{C}}(n)$ are defined as

$$
\left[\delta_{\mathscr{C}}(n)\right]_{p}=\frac{1}{2} \mathscr{C}_{p}+\frac{n+2}{2(n+1)} \inf \left\{\mathscr{C}(\mathscr{L}) \mid \mathscr{L}: \text { a hyperplane of } T_{p} \mathscr{M}\right\}
$$

and

$$
\left[\widehat{\delta}_{\mathscr{C}}(n)\right]_{p}=2 \mathscr{C}_{p}-\frac{2 n+1}{2(n+1)} \sup \left\{\mathscr{C}(\mathscr{L}) \mid \mathscr{L}: \text { a hyperplane of } T_{p} \mathscr{M}\right\} .
$$

Definition 2. A point $p \in \mathscr{M}$ is said to be an invariantly quasi-umbilical point if there exist $2 m-n$ orthogonal unit normal vector $\left\{E_{n+2}, \ldots, E_{2 m+1}\right\}$ such that the shape operator with respect to all directions $E_{r}$ have an eigenvalue of multiplicity $n$ and that for each $E_{r}$ the distinguished eigendirection is the same. The submanifold $\mathcal{M}$ is said to be an invariantlyquasiumbilical submanifold if each of its points is an invariantly quasi-umbilical point.

The following lemmas play a key role in the proof of our theorem:

Lemma 1 ([19]). Let $\vartheta=\left\{\left(x_{1}, x_{2}, \ldots, x_{n}\right) \in \mathbb{R}^{n}: x_{1}+x_{2}+\cdots+x_{n}=k\right\}$ be a hyperplane of $\mathbb{R}^{n}$ and $f: \mathbb{R}^{n} \rightarrow \mathbb{R}$ a quadratic form given by

$$
f\left(x_{1}, x_{2}, \ldots, x_{n}\right)=a \sum_{i=1}^{n-1}\left(x_{i}\right)^{2}+b\left(x_{n}\right)^{2}-2 \sum_{1 \leq i<j \leq n} x_{i} x_{j}, a>0, b>0 .
$$

Then, by the constrained extremum problem, $f$ has a global solution as follows,

provided that

$$
\begin{aligned}
& x_{1}=x_{2}=\cdots=x_{n-1}=\frac{k}{a+1}, \\
& x_{n}=\frac{k}{b+1}=\frac{k(n-1)}{(a+1) b}=(a-n+2) \frac{k}{a+1},
\end{aligned}
$$

$$
b=\frac{n-1}{a-n+2}
$$

Lemma 2 ([15]). Let $N$ be a Riemannian submanifold of Riemannian manifold $(M, G)$, where $g$ is the metric induced on $N$ by $G$ and $f: N \rightarrow \mathbb{R}$ be a differentiable function. If $x_{0} \in N$ is the solution of the constrained extremum problem $\min _{x \in N} f(x)$, then

(i) $(\operatorname{gradf})\left(x_{0}\right) \in T_{x_{0}}^{\perp} N$;

(ii) the bilinear form $A: T_{x_{0}} N \times T_{x_{0}} N \rightarrow \mathbb{R} ; A(X, Y)=\operatorname{Hess}_{f}(X, Y)+G\left(h(X, Y),(\operatorname{grad} f)\left(x_{0}\right)\right)$ is positive semidefinite, where $h$ is the second fundamental form of $N$ in $M$.

Now we are ready to prove the main theorem of the paper. This is as follows:

Theorem 1. Let $M$ be an $(n+1)$-dimensional bi-slant submanifold $\mathscr{M}$ of a generalized Sasakian space form $\overline{\mathscr{M}}\left(f_{1}, f_{2}, f_{3}\right)$ of dimension $(2 m+1)$ and dimensions of $D_{\theta_{1}}$ and $D_{\theta_{2}}$ are $2 n_{1}$ and $2 n_{2}$, respectively. Then 
(i) The normalized $\delta$-Casorati curvature $\delta_{\mathscr{C}}(n)$ satisfies

$$
\rho \leq \delta_{\mathscr{C}}(n)+f_{1}+\frac{6 f_{2}}{n(n+1)}\left(n_{1} \cos ^{2} \theta_{1}+n_{2} \cos ^{2} \theta_{2}\right)-\frac{2 f_{3}}{n+1}\left\|\xi_{1}\right\|^{2}
$$

(ii) The normalized $\delta$-Casorati curvature $\widehat{\delta}_{\mathscr{C}}(n)$ satisfies

$$
\rho \leq \widehat{\delta}_{\mathscr{C}}(n)+f_{1}+\frac{6 f_{2}}{n(n+1)}\left(n_{1} \cos ^{2} \theta_{1}+n_{2} \cos ^{2} \theta_{2}\right)-\frac{2 f_{3}}{n+1}\left\|\xi_{1}\right\|^{2} .
$$

Moreover, the equalities hold in the relations (11) and (12) if and only if $\mathcal{M}$ is an invariantly quasi-umbilical submanifold with the flat normal connection in $\overline{\mathscr{M}}\left(f_{1}, f_{2}, f_{3}\right)$ such that with some orthonormal tangent frame $\left\{E_{1}, \ldots, E_{n+1}\right\}$ of $T \mathscr{M}$ and orthonormal normal frame $\left\{E_{n+2}, \ldots, E_{2 m+1}\right\}$ of $T^{\perp} \mathscr{M}$, the shape operator $\mathscr{S}_{r}, r \in\{n+2, \ldots, 2 m+1\}$, respectively, take the following form:

$$
\mathscr{S}_{n+2}=\left(\begin{array}{cccccc}
d & 0 & 0 & \ldots & 0 & 0 \\
0 & d & 0 & \ldots & 0 & 0 \\
0 & 0 & d & \ldots & 0 & 0 \\
\vdots & \vdots & \vdots & \ddots & \vdots & \vdots \\
0 & 0 & 0 & \ldots & d & 0 \\
0 & 0 & 0 & \ldots & 0 & 2 d
\end{array}\right), \quad \quad \mathscr{S}_{n+3}=\cdots=\mathscr{S}_{2 m+1}=0
$$

and

$$
\mathscr{S}_{n+2}=\left(\begin{array}{cccccc}
2 d & 0 & 0 & \ldots & 0 & 0 \\
0 & 2 d & 0 & \ldots & 0 & 0 \\
0 & 0 & 2 d & \ldots & 0 & 0 \\
\vdots & \vdots & \vdots & \ddots & \vdots & \vdots \\
0 & 0 & 0 & \ldots & 2 d & 0 \\
0 & 0 & 0 & \ldots & 0 & d
\end{array}\right), \quad \mathscr{S}_{n+3}=\ldots=\mathscr{S}_{2 m+1}=0
$$

Proof. Let $\left\{E_{1}, \ldots, E_{n+1}\right\}$ and $\left\{E_{n+2}, \ldots, E_{2 m+1}\right\}$ be the orthonormal basis of $T \mathscr{M}$ and and $T^{\perp} \mathscr{M}$, respectively, at any point $p \in M$. Putting $X=W=E_{i}, Y=Z=E_{j}$ into (3) and considering $i \neq j$, then we have

$$
\begin{aligned}
\sum_{i, j=1}^{n+1} R\left(E_{i}, E_{j}, E_{j}, E_{i}\right)= & \sum_{i, j=1}^{n+1}\left\{f_{1}\left\{g\left(E_{j}, E_{j}\right) g\left(E_{i}, E_{i}\right)-g\left(E_{i}, E_{j}\right) g\left(E_{j}, E_{i}\right)\right\}\right. \\
& +f_{2}\left\{g\left(E_{i}, \phi E_{j}\right) g\left(\phi E_{j}, E_{i}\right)-g\left(\phi E_{i}, E_{i}\right) g\left(E_{j}, \phi E_{j}\right)\right. \\
& \left.+2 g\left(E_{i}, \phi E_{j}\right) g\left(E_{i}, \phi E_{j}\right)\right\}+f_{3}\left\{\eta\left(E_{i}\right) \eta\left(E_{j}\right) g\left(E_{i}, E_{i}\right)\right. \\
& \left.\left.-\eta\left(E_{j}\right) \eta\left(E_{j}\right) g\left(E_{i}, E_{i}\right)+\eta\left(E_{i}\right) \eta\left(E_{j}\right) g\left(E_{i}, E_{j}\right)-\eta\left(E_{i}\right) \eta\left(E_{i}\right) g\left(E_{j}, E_{j}\right)\right\}\right\} .
\end{aligned}
$$

From this and together with Gauss equation, we have [16]

$$
2 \tau(p)=n(n+1) f_{1}+6 f_{2}\left(n_{1} \cos ^{2} \theta_{1}+n_{2} \cos ^{2} \theta_{2}\right)-2 n f_{3}\left\|\xi_{1}\right\|^{2}+(n+1)^{2}\|\not \mathscr{C}\|^{2}-(n+1) \mathscr{C},
$$

where we have used (10). 
We define now the following function, denoted by $\mathscr{Q}$, which is a quadratic polynomial in the components of the second fundamental form:

$$
\begin{aligned}
\mathscr{Q}= & \frac{1}{2} n(n+1) \mathscr{C}+\frac{1}{2}(n+2) \mathscr{C}(\mathscr{L})-2 \tau(p)+n(n+1) f_{1} \\
& +6 f_{2}\left(n_{1} \cos ^{2} \theta_{1}+n_{2} \cos ^{2} \theta_{2}\right)-2 n f_{3}\left\|\xi_{1}\right\|^{2}
\end{aligned}
$$

where $\mathscr{L}$ is a hyperplane of $T_{p} \mathscr{M}$. We can assume without loss of generality that $\mathscr{L}$ is spanned by $\left\{E_{1}, \ldots, E_{n}\right\}$. Then we have

$$
\mathscr{Q}=\frac{n+2}{2} \sum_{r=n+2}^{2 m+1} \sum_{i, j=1}^{n+1}\left(h_{i j}^{r}\right)^{2}+\frac{n+2}{2} \sum_{r=n+2}^{2 m+1} \sum_{i, j=1}^{n}\left(h_{i j}^{r}\right)^{2}-\sum_{r=n+2}^{2 m+1}\left(\sum_{i, j=1}^{n+1} h_{i j}^{r}\right)^{2} .
$$

Now we can easily derive that

$$
\begin{aligned}
\mathscr{Q}= & \sum_{r=n+2}^{2 m+1} \sum_{i=1}^{n}\left[(n+1)\left(h_{i i}^{r}\right)^{2}+(n+2)\left(h_{i n+1}^{r}\right)^{2}\right] \\
& +\sum_{r=n+2}^{2 m+1}\left[2(n+2) \sum_{i<j=1}^{n}\left(h_{i j}^{r}\right)^{2}-2 \sum_{i<j=1}^{n+1} h_{i i}^{r} h_{j j}^{r}+\frac{n}{2}\left(h_{n+1 n+1}^{r}\right)^{2}\right] . \\
\geq & \sum_{r=n+2}^{2 m+1} \sum_{i=1}^{n}\left[(n+1)\left(h_{i i}^{r}\right)^{2}+\sum_{r=n+2}^{2 m+1}\left[-2 \sum_{i<j=1}^{n+1} h_{i i}^{r} h_{j j}^{r}+\frac{n}{2}\left(h_{n+1 n+1}^{r}\right)^{2}\right] .\right.
\end{aligned}
$$

For $r=n+2, \ldots, 2 m+1$, let us take the quadratic form $\phi_{r}: \mathbb{R}^{n+1} \rightarrow \mathbb{R}$, defined by

$$
\phi_{r}\left(h_{11}^{r}, \ldots, h_{n+1 n+1}^{r}\right)=\sum_{r=n+2}^{2 m+1} \sum_{i=1}^{n}\left[(n+1)\left(h_{i i}^{r}\right)^{2}+\sum_{r=n+2}^{2 m+1}\left[-2 \sum_{i<j=1}^{n+1} h_{i i}^{r} h_{j j}^{r}+\frac{n}{2}\left(h_{n+1 n+1}^{r}\right)^{2}\right] .\right.
$$

and the constrained extremum problem $\min \phi_{r}$ subject to the component of trace $\mathscr{H}$,

$$
\theta: h_{11}^{r}+\cdots+h_{n+1 n+1}^{r}=k^{r}
$$

where $k^{r}$ is a real constant.

Comparing (18) with the quadratic function in Lemma 1, we find that

$$
a=\frac{n^{2}+n+2}{2 n}, b=\frac{n}{2} .
$$

Therefore, we can find the critical point $\left(h_{11}^{r}, \ldots, h_{n+1 n+1}^{r}\right)$ :

$$
\begin{gathered}
h_{11}^{r}=h_{22}^{r}=\cdots=h_{n n}^{r}=\frac{k^{r}}{a+1}=\frac{2 n k^{r}}{(n+1)(n+2)}, \\
h_{n+1 n+1}^{r}=\frac{k^{r}}{b+1}=\frac{2 k^{r}}{n+2} .
\end{gathered}
$$

Now here we use Lemma 2 and for this, we fix an arbitrary point $x_{0} \in \theta$. The bilinear form

$$
A: T_{x_{0}} \theta \times T_{x_{0}} \theta \rightarrow \mathbb{R}
$$


is defined by

$$
A(X, Y)=\operatorname{Hess}_{\phi_{r}}(X, Y)+\left\langle\hbar(X, Y),\left(\operatorname{grad} \phi_{r}\right)\left(x_{0}\right)\right\rangle
$$

where $\hbar$ is the second fundamental form of $\theta$ in $\mathbb{R}^{n+1}$ and $\langle$,$\rangle is the standard inner product on$ $\mathbb{R}^{n+1}$. So, we have the following:

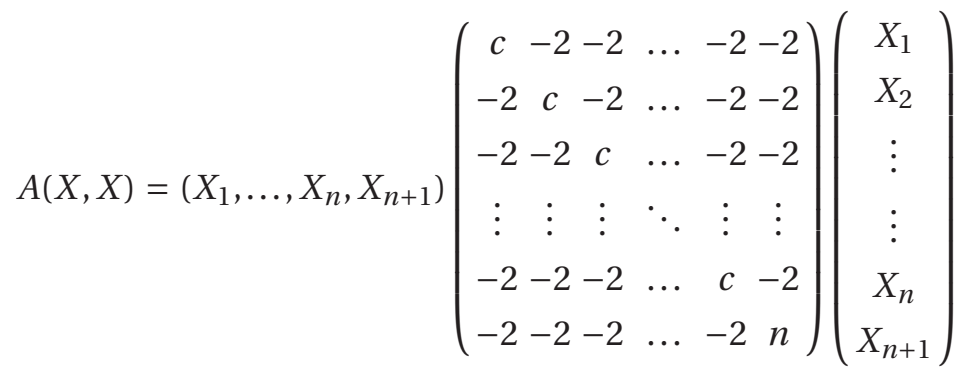

$$
\begin{aligned}
& \geq 0 \text {, }
\end{aligned}
$$

where $c=\frac{n^{2}+n+2}{n}$. Thus, the point $\left(h_{11}^{r}, \ldots, h_{n+1 n+1}^{r}\right)$ (see (19)) is a global minimum point. From relation (17) and (19), we get $\mathscr{Q} \geq 0$ and hence we have

$$
2 \tau(p) \leq \frac{1}{2} n(n+1) \mathscr{C}+\frac{1}{2}(n+2) \mathscr{C}(\mathscr{L})+n(n+1) f_{1}+6 f_{2}\left(n_{1} \cos ^{2} \theta_{1}+n_{2} \cos ^{2} \theta_{2}\right)-2 n f_{3}\left\|\xi_{1}\right\|^{2},
$$

whereby, we obtain

$$
\rho \leq \frac{1}{2} \mathscr{C}+\frac{1}{2}\left(\frac{(n+2)}{n(n+1)}\right) \mathscr{C}(\mathscr{L})+f_{1}-\frac{2 f_{3}}{n+1}\left\|\xi_{1}\right\|^{2}+\frac{6 f_{2}}{n(n+1)}\left(n_{1} \cos ^{2} \theta_{1}+n_{2} \cos ^{2} \theta_{2}\right) .
$$

By the definition of $\delta_{\mathscr{C}}(n)$, we can obtain our desired inequality (11). Moreover, the equality sign holds if and only if

$$
h_{i j}^{r}=0, \forall i, j \in\{1, \ldots, n+1\}, i \neq j, r \in\{n+2, \ldots, 2 m+1\}
$$

and

$$
h_{n+1 n+1}=2 h_{11}^{r}=\cdots=2 h_{n n}^{r}, \forall r \in\{n+2, \ldots, 2 m+1\}
$$

From (20) and (21), we conclude that the equality sign holds in the inequality (11) if and only if the submanifold $\mathscr{M}$ is invariantly quasi-umbilical with trivial normal connection in $\mathscr{M}$, such that with respect to suitable orthonormal tangent and normal orthonormal frames, the shape operators take the form of (13).

In the same manner, we can easily obtain another inequality (12) and also show that the equality at all points characterizes the invariantly quasi-umbilical submanifolds.

Remark 3. Theorem 1 shows that the normalized scalar curvature for bi-slant submanifold of generalized Sasakian space form is bounded above by the normalized Casorati curvatures $\delta_{\mathscr{C}}(n)$ and $\widehat{\delta}_{\mathscr{C}}(n)$. 
Remark 4. With similar proof of Theorem 1, we can show that the normalized scalar curvature is bounded above by the normalized Casorati curvatures $\delta_{\mathscr{C}}(n)$ and $\widehat{\delta}_{\mathscr{C}}(n)$ when ambient space form is, respectively, [16]

(i) Sasakian space form.

(ii) Kenmotsu space form.

(iii) cosymplectic space form.

\section{Acknowledgement}

The author wishes to express her sincere thanks to the referee(s) for valuable comments and suggestions towards the improvement of the present paper.

\section{References}

[1] P. Alegre, D. E. Blair and A. Carriazo, Generalized Sasakian-space-forms, Israel J. Math., 141 (2004), $157-183$.

[2] J. L. Cabrerizo, A. Carriazo, L. M. Fernandez and M. Fernandez, Semi-slant submanifolds of a Sasakian manifold, Geometria Dedicata, 78 (1999), 183-199.

[3] J. L. Cabrerizo, A. Carriazo, L. M. Fernandez and M. Fernandez, Slant submanifolds in Sasakian manifolds, Glasgow Math. J., 42 (2000), 125-138.

[4] B. Y. Chen, Relationship between Ricci curvature and shape operator for submanifolds with arbitrary codimensions, Glasgow. Math. J., 41 (1999), 33-41.

[5] B. Y. Chen, Some pinching and classification theorems for minimal submanifolds, Arch. Math., 60 (1993), 568-578.

[6] S. Decu, S. Haesen and L. Verstralelen, Optimal inequalities involving Casorati curvatures, Bull. Transylv. Univ. Brasov, Ser. B, 14 (2007), 85-93.

[7] S. Decu, S. Haesen and L. Verstralelen, Optimal inequalities characterizing quasi-umbilical submanifolds, J. Inequalities Pure. Appl. Math., 9 (2008), Article ID 79, 7pp.

[8] V. Ghisoiu, Inequalities for thr Casorati curvatures of the slant submanifolds in complex space forms, Riemannian geometry and applications. Proceedings RIGA 2011, ed. Univ. Bucuresti, Bucharest (2011), 145-150.

[9] V. A. Khan and M. A. Khan, Pseudo-slant submanifolds of a Sasakian manifold, Indian J. Pure Appl. Math., 38 (2007), 31-42.

[10] D. Kowalczyk, Casorati curvatures, Bull. Transilvania Univ. Brasov Ser. III, 50(1) (2008), 2009-2013.

[11] C. W. Lee, J. W. Lee and G. E. Vilcu, Optimal inequalities for the normalized $\delta$-Casorati curvatures of submanifolds in Kenmotsu space forms, Advances in Geometry, 17 (2017), in press; doi:10.1515/advgeom-2017-0008.

[12] C. W. Lee, D. W. Yoon and J. W. Lee, Optimal inequalities for the Casorati curvatures of submanifolds of real space forms endowed with semi-symmetric metric connections, J. Inequal. Appl., (2014), 2014:327, 9 pp. MR 3344114.

[13] J. W. Lee and G. E. Vilcu, Inequalities for generalized normalized $\delta$-Casorati curvatures of slant submanifolds in quaternion space forms, Taiwanese J. Math., 19 (2015), 691-702.

[14] C. W. Lee, J. W. Lee, G. E. Vilcu and D. W. Yoon, Optimal inequalities for the Casorati curvatures of the submanifolds of generalized space form endowed with semi-symmetric metric connections, Bull. Korean Math. Soc., 52 (2015), 1631-1647.

[15] T. Oprea, Optimization methods on Riemannian submanifolds, An. Univ Bucur. Mat., 54 (2005), $127-136$.

[16] A. N. Siddiqui and M. H. Shahid, A Lower Bound of Normalized Scalar Curvature for Bi-Slant Submanifolds in Generalized Sasakian Space Forms using Casorati Curvatures, Acta Math. Univ. Comenianae, LXXXVII(1) (2018), 127-140. 
[17] A. N. Siddiqui, Upper Bound Inequalities for $\delta$-Casorati Curvatures of Submanifolds in Generalized Sasakian Space Forms Admitting a Semi-Symmetric Metric Connection, Inter. Elec. J. Geom., 11(1)(2018), 57-67.

[18] M. Su et al., Some inequalities for submanifolds in a Riemannian manifold of nearly quasi-constant curvature, Filomat, 31(8) (2017), 2467-2475; doi:10.2298/FIL1708467S.

[19] M. M. Tripathi, Inequalities for algebraic Casorati curvatures and their applications, Note Mat., 37 (2017), 161-186.

[20] L. Verstralelen, Geometry of submanifolds I, The first Casorati curvature indicatrices, Kragujevac J. Math., 37 (2013), 5-23.

[21] L. Verstralelen, The geometry of eye and brain, Soochow J. Math., 30 (2004), 367-376.

[22] K. Yano and M. Kon, Differential geometry of CR-submanifolds, Geometria Dedicata, 10 (1981), 369-391.

[23] K. Yano and M. Kon, Structures on Manifolds, Worlds Scientific, Singapore, 1984.

[24] P. Zhang and L. Zhang, Casorati inequalities for submanifolds in a Riemannian manifold of quasi-constant curvature with a semi-symmetric metric connection, Symmetry, 8(4) (2016), 19; doi:10.3390/sym8040019.

Department of Mathematics, Jamia Millia Islamia, New Delhi - 110025, India.

E-mail: aliyanaazsiddiqui9@gmail.com 